\title{
Gastric Volvulus and Attendant Complications
}

\author{
Henry Odhiambo Owuor, Patrick Chege
}

School of Medicine, Moi University

Correspondence to: Dr. Henry Owuor, PO Box 3900-30100 Eldoret, Kenya; email: owuorkembo@gmail.com

\section{Summary}

The diagnosis of a hiatal hernia, a gastric volvulus or its attendant complications, can be easily missed because evaluation of acute abdominal pain can be difficult, particularly in resource-limited settings. Diagnosis of a gastric volvulus is even harder in the pediatric population because its peak occurrence is in the fifth decade. Hiatal hernia (and gastric volvulus) can be found incidentally in chest radiographs that are requested routinely or in suspected pulmonary pathology. The gold standard diagnostic tool for a gastric volvulus is a barium swallow. The optimal treatment method is open laparotomy with detorsion, and prevention of recurrence with anterior gastropexy. We present a case of an 8-year-old girl who had a diagnosis of hiatal/paraesophageal hernia with a gastric volvulus complicated by ischemia, necrosis, perforation and collapse of the left lung. A complete history and a thorough evaluation of the sick-looking patient are paramount in diagnosing life-threatening conditions like gastric volvulus.

Key words: Gastric volvulus, Complications, Paraesophageal hernia, Borchardt's triad

Ann Afr Surg. 2019; 16(1):46-48

DOI: http://dx.doi.org/10.4314/aas.v16i1.12

\section{Conflicts of Interest: None}

\section{Funding: None}

(C) 2019 Author. This work is licensed under the Creative Commons Attribution 4.0 International License.

\section{Introduction}

Gastric volvulus is life threatening and needs timely diagnosis and management (1). Its peak occurrence is in the fifth decade, and it has no preference for any sex $(2,3)$. The major complication of gastric volvulus is gastric outlet obstruction which may be acute, intermittent or chronic (4). In addition to obstruction, it may cause strangulation, necrosis, ischemia, perforation and hypovolemic shock (4). Being a rare condition, it has a high mortality rate, between 30 and $50 \%$, when it complicates $(2,5)$. We present a fatal case of gastric volvulus to emphasize that clinicians should have a high index of suspicion for this condition even though it is rare, so as to avert its high case fatality rate.

\section{Case Presentation}

An 8-year-old girl on follow-up for peptic ulcer disease was admitted with exacerbation of the peptic ulcer disease for 4 days. She had had intermittent epigastric pain for the past 6 years but this had worsened over the four days before her admission. This pain was associated with nausea and post-prandial vomiting; ahe was vomiting everything she ate or drunk. The vomitus then progressed to coffeeground. The pain was not relieved by intravenous ranitidine or buscopan, and the vomiting was also not relieved by intravenous metoclopramide. She was restless, assuming the knee-chest position in bed and crying in pain. There was also hotness of body. On review of systems, there was no headache, cough, chest-pains, difficulty in breathing, diarrhea, urinary symptoms, wasting or drenching night sweats. She had had two previous admissions for malaria but had no history of blood transfusion or surgery. Her infancy was uneventful with no developmental delays and her vaccination was up to date. The patient was in respiratory distress, tachypnoiec (60 breaths per minute) and in shock (cold peripheries, weak pulse, heart rate of 164 beats per minute with saturations unrecordable peripherally). Her general exam was unremarkable, but she had reduced air entry over the left lung with no areas of dullness. Abdominal examination was unremarkable except for a mildly enlarged, smooth liver $6 \mathrm{~cm}$ below the costal margin. There was no peritonism.

A blood slide was negative for malaria parasites and a sickling test and $H$. pylori antibody test were also negative. Her random blood sugar was $5.4 \mathrm{mmol} / \mathrm{L}$. Two previous abdominal ultrasound scans showed a dilated gall bladder and a lot of epigastric gas, suggesting gastritis. The patient collapsed and died on her way to the radiology department for a chest radiograph.

During the post-mortem, a distended stomach was found in left hemithorax in organoaxial volvulus of $360^{\circ}$. It had a large gangrenous area, atrophied (smooth) mucosa with coffee ground liquid in the stomach. There were also two 
perforations along the greater curvature and hemorrhagic fluid in the left side of the chest cavity. Both lobes of the left lung had collapsed. In the abdomen was a gangrenous $5-\mathrm{cm}$ length of ileum, approximately $25 \mathrm{~cm}$ from the ligament of Treitz. The gallbladder was enlarged, and the liver also appeared enlarged. No nodules or other gross pathology were seen. Other body parts were normal.

\section{Discussion}

Gastric volvulus is a rare and therefore easily forgotten disease (1). It is the rotation of the stomach along its long or short axis or both, creating either a gastric outlet obstruction or a closed loop (2). Its incidence and prevalence are unknown (1). Its incidence however peaks in the 5th decade of life, and children constitute only $10-20 \%$ of reported cases (2). There is no association with sex while race associations have not been documented (2).

Gastric volvulus can be either primary or secondary. Primary (idiopathic), making up a third of cases, is usually due to abnormalities of the gastric ligaments which anchor the stomach and prevent rotation, whereas secondary gastric volvulus is due to other anatomic abnormalities like hiatal or paraesophageal hernia, diaphragmatic eventration, phrenic nerve paralysis or congenital diaphragmatic hernia in children (3). Depending on the axis of rotation, gastric volvulus can be classified as organoaxial rotation or mesenteroaxial rotation, which is rotation of the stomach along its long axis or through a perpendicular line connecting the greater and lesser curvatures of the stomach respectively (3). A rotation greater than $180^{\circ}$ causes signs of acute gastric outlet obstruction (accounting for $40 \%$ of cases) whereas those with lesser degrees of rotation have signs of partial gastric obstruction that can be intermittent and chronic (4). The latter can have a superimposition of acute on chronic (4). A history of reflux points to the presence of a hernia.

This condition may present as an acute abdomen or as a chronic upper abdominal or retrosternal discomfort (5). In acute gastric volvulus, epigastric pain, vomiting that may be unproductive and the inability to pass a nasogastric tube (Borchardt's triad) may be present in nearly $70 \%$ of cases (1). Additional features include minimal abdominal findings when the stomach is in the thorax, a gas-filled viscus in the lower chest or upper abdomen on chest radiograph (in a paraesophageal hernia) and obstruction at the site of the volvulus, shown by upper gastrointestinal (GI) series (6). The rotation can cause venous congestion and mucosal ischemia. In addition, gastric infarction and MalloryWeiss tears can cause hematemesis, acute cardiopulmonary distress or shock (3). Strangulation and necrosis are the most life-threatening complications of acute gastric volvulus. Gastric perforation may ensue. The three commonly cause sepsis and cardiovascular collapse (6).

Plain radiography should be the initial diagnostic sign when an acute gastric volvulus is suspected. A single large spherical gas bubble located in the upper abdomen or chest with an air-fluid level will be seen on the antero-posterior view (7). A barium swallow (or upper GI series) has been the gold standard in diagnosing gastric volvulus (8). However, a CT scan will show the anatomic position of the stomach and aid the identification of anatomic abnormalities associated with secondary gastric volvulus, e.g. diaphragmatic abnormalities (9). Endoscopy misses nearly three-quarters of these cases and is therefore unreliable for a diagnosis of gastric volvulus (10).

The goal of management is to improve gastric perfusion and reduce the risk for ischemia and necrosis. This can be achieved by gastric decompression using a naso-gastric tube, or endoscopically $(1,3)$. The gold standard of management however is open laparotomy with detorsion and anterior gastropexy to prevent recurrences (1). In fact, immediate surgery is vital in acute volvulus to prevent vascular compromise and other complications (5). Correcting the associated abnormalities forms part of the definitive treatment if the volvulus is secondary (11).

\section{Conclusion}

Acute abdominal pain is a common presentation and clinicians must consider multiple diagnoses, especially lifethreatening conditions like gastric volvulus (12). Mortality from acute gastric volvulus can be as high as $30-50 \%$ (13). However, this rate can be reduced to between 15 and $20 \%$ with rapid diagnosis and modern treatment (14). Although a rare disease, gastric volvulus has a high mortality rate. The primary care physician should be aware of the tell-tale signs and institute early management. Identification of Borchardt's triad coupled with a plain radiograph and a high index of suspicion may be diagnostic in resource-poor settings. There is no need for over-reliance on modern diagnostic modalities. In fact, the modern physician should be humbled that, despite diagnostic and therapeutic advances like CT, ultrasonography, and laparoscopy, the misdiagnosis rate of even the most common surgical emergencies like acute appendicitis has changed little over time (15). Therefore, one must obtain as complete a history as possible as this is the cornerstone of an accurate diagnosis. In addition, sicklooking patients with abdominal pain are of great concern and must be thoroughly evaluated for potentially lifethreatening causes. Patients with reflux should also have a 
thorough history, physical examination and diagnostic work up to rule in or rule out chronic gastric volvulus or a hernia.

\section{Acknowledgements}

The authors wish to acknowledge the family of the deceased for consent to perform the post-mortem and to allow an academic discourse following their kin's demise. The authors also acknowledge the department of Family Medicine, Moi University, as it actively contributed in performing the post-mortem and giving direction to the discussion of the case. We also thank the medical superintendent and the mortuary department in-charge of Webuye County Hospital for allowing the post-mortem to be conducted at no cost at the Webuye County Hospital mortuary.

\section{References}

1. Chau B, Dufel S. Gastric volvulus. EMJ. 2007; 24(6):446-7.

2. Wu MH, Chang $\mathrm{YC}, \mathrm{Wu} \mathrm{CH}$. Acute gastric volvulus: A rare but real surgical emergency. Am J Emerg Med. 2011; 28:118. e5.

3. Rashid F, Thangarajah T, Mulvey D. A review article on gastric volvulus: a challenge to diagnosis and management. Int J Surg. 2010; 8:18.

4. Shivanand G, Seema S, Srivastava S, et al. Gastric volvulus: Acute and chronic presentation. Clin Imaging. 2003; 27:265.
5. Cardile AP, Heppner DS. Gastric volvulus, Borchardt's triad, and endoscopy: A rare twist. Hawaii Med J. 2011; 70(4):80-2.

6. Carter R, Brewer LA, Hinshaw DB. Acute gastric volvulus: A study of 25 cases. Am J Surg. 1980; 140:99-104.

7. Oh SK, Han BK, Levin TL, et al. Gastric volvulus in children: The twists and turns of an unusual entity. Pediatr Radiol. 2008; 38:297.

8. Kohli A, Vij A, Azad T. Intrathoracic gastric volvulus-Acute and chronic presentation. J Indian Med Assoc. 1997; 95:522-3.

9. Peterson CM, Anderson JS, Hara AK, et al. Volvulus of the gastrointestinal tract: appearances at multi-modality imaging. Radiographics. 2009; 29(5):1281-93.

10. Hsu YC, Perng CL, Chen CK, et al. Conservative management of chronic gastric volvulus: 44 cases over 5 years. World J Gastroenterol. 2010; 16:4200.

11. Lee JS, Park JW, Sohn JW, et al. Organo-axial volvulus of the stomach with diaphragmatic eventration. Korean J Intern Med. 2000;15(2):127-30.

12. Macaluso CR, Mcnamara RM. Evaluation and management of acute abdominal pain in the Emergency Department. Int J of Gen Med. 2012; 5:789-797.

13. Smith RJ. Volvulus of the stomach. JAMA.1983; 75:393-6.

14. Rat OHP, Christophe M, Friedman S, et al. Surgical results of intrathoracic gastric volvulus complicating hiatal hernia. $\mathrm{Br} \mathrm{J}$ Surg. 1990; 77:1379-81.

15. Flum DR, Morris A, Koepsell T, et al. Has misdiagnosis of appendicitis decreased over time? JAMA. 2001; 286:1748-53. 